\section{$\underset{\substack{\text { hommes } \\ \text { \& migrations }}}{ }$}

\section{Hommes \& migrations}

Revue française de référence sur les dynamiques

migratoires

$1314 \mid 2016$

Migrations chinoises et générations

\title{
Le modèle national à la lumière de parcours individuels marseillais
}

\section{Marie Beschon}

\section{OpenEdition \\ Journals}

\section{Édition électronique}

URL : http://journals.openedition.org/hommesmigrations/3654

DOI : 10.4000/hommesmigrations.3654

ISSN : 2262-3353

\section{Éditeur}

Musée national de l'histoire de l'immigration

\section{Édition imprimée}

Date de publication : 1 avril 2016

Pagination : 137-140

ISBN : 978-2-919040-35-3

ISSN : $1142-852 X$

\section{Référence électronique}

Marie Beschon, «Le modèle national à la lumière de parcours individuels marseillais », Hommes \& migrations [En ligne], 1314 | 2016, mis en ligne le 19 septembre 2016, consulté le 14 septembre 2020. URL : http://journals.openedition.org/hommesmigrations/3654 


\title{
LA REPRÉSENTATION DES IMMIGRANTS \\ DES IMMIGRANTS EN REPRÉSENTATION ? LE CAS DES ASIATIQUES, VIETNAMIENS EN PARTICULIER
}

\author{
Par LIÊM-KHÊ LUGUERN, docteure en histoire, IRIS-EHESS.
}

a société indochinoise n'est pas un bloc'. Ses Ldisparités, antagonismes sociaux, politiques trouvent leur prolongement dans l'immigration en France. C'est ce qu'ont montré les travaux réalisés depuis les années 1980, lesquels, à l'exception de la thèse de Lê-Huu-Khoa sur les Vietnamiens en France ${ }^{2}$, sont circonscrits à des épisodes migratoires spécifiques : travailleurs de la Grande Guerre $^{3}$, de la Seconde Guerre mondiale ${ }^{4}$, rapatriés d'Indochine ${ }^{5}$, réfugiés des années $1970^{6}$. Plus que l'invisibilité des Asiatiques dans leur ensemble, c'est surtout l'invisibilité des employés et des ouvriers dans cette immigration qui est porteuse de questions? Cette invisibilité est au cœur des représentations collectives sur les Asiatiques jusqu'à la fin du siècle dernier, représentations forgées par des immigrants eux-mêmes en représentation : ce que l'on voit n'est-il pas ce qui est donné à voir?

\section{Tous des Chinois?}

Tous les immigrés asiatiques le savent : ils sont tous "chinois" aux yeux des Français. Si les Chinois (de nationalité et/ou d'origine) forment la majo- rité des immigrants asiatiques aujourd'hui, ce fait est récent. Selon les sources et les critères, les Chinois seraient aujourd'hui proches de 500000 en France soit entre le double et le triple des Vietnamiens, deuxième communauté asiatique ${ }^{8}$. L'arrivée en nombre des Chinois date de la fin des années 1970. Ils forment, en effet, la grande majorité des boat people fuyant le régime mis en place au Vietnam en 1975 où vivait jusque-là une importante communauté chinoise. L'immigration en provenance de l'Indochine est plus ancienne, en raison du passé colonial. Lémigration des Vietnamiens (la grande majorité des émigrants Indochinois) vers la France est, en effet, un processus permanent depuis le début du XXe siècle. Cependant, les regards extérieurs ne font aucunement la différence entre un Cambodgien, un Chinois, un Vietnamien... On peut parler sous cet angle de communauté asiatique dans la mesure où tous les individus qui la composent bénéficient ou subissent les mêmes stéréotypes. Cela permet d'aborder la question des représentations ancrées qui se construisent donc sur un temps long mais aussi leur mutation. L'histoire de cette immigration reflète les soubresauts de l'histoire 


\section{REPÉRAGE}

contemporaine (Guerres mondiales, décolonisation, guerre froide). Jusqu'en 1914, cette immigration reste circonscrite à une centaine d'individus. Avec la Grande Guerre, son effectif passe à 90000 hommes : les travailleurs et tirailleurs recrutés. Le même scénario se reproduit en 1939-1940 pour 27000 hommes. En 1954, à la fin de la guerre d'Indochine, et jusqu'en 1965, les rapatriés d'Indochine sont estimés entre 30 et 35 ooo. Enfin, la dernière grande vague d'immigration correspond à l'épisode des boat people. L'association France terre d'asile estime que 42694 Vietnamiens sont alors accueillis en France au titre de réfugiés (pour 75000 Chinois, issus de l'ex-Indochine). Globalement, la présence des Vietnamiens en France est donc très minoritaire et n'apparaît dans les statistiques de l'Institut national de la statistique et des études économiques (INSEE) qu'en 1975 (Cambodgiens, Laotiens et Vietnamiens représentent alors $0,5 \%$ des étrangers, $2,8 \%$ en

Si l'on regarde de plus près,

les Vietnamiens

n’ont pas toujours été une "communauté protégée". 1982 et 3,1\% en 1990).

Au total, jusqu'en 1954, la présence vietnamienne se stabilise autour de 5000 individus, essentiellement jeunes et masculins, soit une présence marginale en comparaison avec d'autres immigrations en France. Elle est multipliée par sept dans les années 1950-1960, par l'arrivée des rapatriés d'Indochine qui s'ajoute au flux numériquement marginal mais continu d'étudiants et de familles aisées fuyant la guerre du Vietnam. Enfin ses effectifs bondissent avec l'arrivée des boat people qui, dans un premier temps, sont formés de ressortissants des couches sociales moyenne et supérieure.

\section{La représentation : un terrain de luttes}

Nous n'avons guère ici l'espace pour nous replonger dans les origines anciennes qui participent à la formation des stéréotypes de l'Asiatique. On peut se reporter pour cela à l'ouvrage le plus complet en la matière, sur le plan factuel : Le Paris Asie ${ }^{9}$ qui rappelle l'attrait et la fascination pour l'Asie depuis... Marco Polo ${ }^{10}$. Les qualificatifs désignant la population de l'Extrême-Orient sont quasi-invariables à travers les siècles : "mystérieuse", "secrète", "silencieuse" hier, elle devient aujourd'hui "polie", "discrète" et "souriante" ; "fumeuse d'opium", "trafiquante" hier, associée aujourd'hui, à "clandestin", "jeu”, "racket", "drogues"; hier "disciplinée", "laborieuse", aujourd'hui "travailleuse", "réussite scolaire et sociale"... Les stéréotypes peuvent se décliner selon des axiologies positives ou négatives et un même stéréotype peut être polarisé positivement ou négativement. C'est le cas de la représentation de l'immigration "laborieuse" et "disciplinée". Associée aux étudiants, elle se décline par "la réussite scolaire", et appliquée aux paysans et travailleurs chinois récemment arrivés, elle renvoie aux ateliers "clandestins" ou "appartementsraviolis" pour reprendre une image choc répandue par les media où la main-d'œuvre travaillent sans relâche et sans renâcler dans des conditions inhumaines pour fournir les commerces de bouche".

Si l'on regarde de plus près, les Vietnamiens n'ont pas toujours été une "communauté protégée". Compte tenu de la faiblesse numérique de

7. De ce point de vue, la récente publication de Solène Granier donne à voir la complexité sociologique de la "communauté vietnamienne", Domestiques Indochinois, Paris, Vendémiaires, 2014, 224 p. 8. Les estimations hautes prennent en compte ceux qui ont gardé la nationalité du pays d'origine, les naturalisés qui, surtout dans le cas des Chinois, ont gardé un lien fort avec celui-ci et également les enfants nés en France. Ces estimations, très difficiles à établir, varient considérablement selon les sources et les critères retenus. 9. Pascal Blanchard, Éric Deroo, Le Paris Asie. 150 Ans de présence de la Chine, de l'Indochine, du Japon... dans la capitale, Paris, La Découverte, 2004. 10. Ibid 11. L'expression "appartements raviolis" a été introduite à grande échelle par le reportage de J.C. Doria, "Faut-il avoir peur des restaurants asiatiques ?", diffusé dans l'émission Envoyé Spécial, France 2, 2004. Un second volet a été diffusé en 2009 dans la même émission. Le thème a été relayé dans les journaux télévisés. 
cette immigration, le fantasme de l'invasion et du "péril jaune" n’a jamais vraiment fonctionné. En revanche, sur d'autres thèmes, on retrouve les mêmes procédés de mobilisation collective décrits par Gérard Noiriel ${ }^{12}$ : martèlement de préjugés (" Les Indochinois sont fourbes et orgueilleux ") et mise en avant des petits faits de la vie quotidienne qui accentuent et amplifient le dégoût ("Les Indochinois mangent des chiens", "ils ont des dents noires"13).

Les stéréotypes sont donc ambivalents et de ce fait brouillent les représentations. La raison tient dans la pluralité des strates sociales qui composent cette immigration. À la différence d'autres immigrations comme les mineurs polonais ou les OS Algériens, par exemple, les Asiatiques n'ont pas constitué de population socialement homogène sur un temps court. De là, dans un premier temps, l'absence de stigmate. Sa fabrication suppose, en effet, comme le rappelle Gérard Noiriel, la conjugaison d'au moins deux composantes: l'origine et la position sociale ${ }^{14}$. Cependant, au tournant des années 1980, la dernière génération d'immigrants asiatiques apparaît socialement plus homogène et numériquement plus importante. Issue notamment des zones rurales ou des friches industrielles de la Chine, elle est caractérisée par un faible niveau scolaire et de qualification. Elle fournit un réservoir de travailleurs manuels contraints d'accepter des conditions de travail très pénibles, parfois dans la clandestinité. C'est au sein de cette population que l'on trouve les sans-papiers. Cette figure du "sanspapier " est en voie de changer de "couleur". Nous nous trouvons en présence de deux ingrédients qui, conjugués, forment le stigmate : chinois et pauvre ou, autre variante, chinois et clandestin. Dans leur enquête sur le Triangle de Choisy, les sociologues Michelle Guillon et Isabelle Taboada
Leonetti montrent que d'année en année depuis les années 1980, "le statut social, le niveau scolaire et la connaissance du français des réfugiés qui arrivent en France diminuent ${ }^{15}$ ". De même, le mythe de la bonne intégration professionnelle ne résiste pas à l'examen des données : le taux de chômage des Vietnamiens au recensement de 1990 est près de $31 \%$, soit le plus élevé pour l'ensemble des étrangers naturalisés ${ }^{16}$. C'est aussi ce qui explique le tournant dans les stéréotypes vis-à-vis Pour les Vietnamiens, cela a commencé "positivement" à la fin des années 1970, avec l'action très médiatisée de Bernard Kouchner recueillant les réfugiés en mer de Chine. de l'Asiatique au début des années 1980 : il correspond au retour du discours national-sécuritaire que Gérard Noiriel date des années 1981-1983. En ce domaine, il n'y a donc pas non plus de spécificité pour la "communauté asiatique", le discours qui porte sur elle obéit, selon Noiriel, à "une nouvelle matrice discursive, adaptée aux normes qui régissent aujourd'hui l'industrie de la communication ${ }^{17}$.

\section{La communication des stéréotypes}

Pour les Vietnamiens, cela a commencé "positivement" à la fin des années 1970, avec l'action très médiatisée de Bernard Kouchner recueillant les réfugiés en mer de Chine. Selon le sociologue Pierre-Jean Simon, cette image positive est alimentée par l'histoire post-coloniale (guerre antiaméricaine, engouement pour le bouddhisme, exode des réfugiés après 1975) bien plus que par I'histoire coloniale de l'Indochine française ${ }^{18}$. C'est là une différence avec l'histoire des migrants d'Afrique du nord coloniale. Mais, à partir des 


\section{REPÉRAGE}

années 1980, Michelle Guillon et Isabelle Taboada Leonetti montrent que la valeur négative a été peu à peu développée par la presse. En 1982, surgit brutalement le thème de la clandestinité et de l'illégalité : les termes "clandestin", "drogue”, "trafic", "héroïne" devancent désormais "solidarité", "sourire", "réussite". On peut situer en 1980 le point de départ de la réactivation des vieux fantasmes, avec les rumeurs largement médiatisées du faible nombre de décès d'immigrants asiatiques enregistrés dans le XIII arrondissement de Paris. Vient ensuite dans les années 1990 et 2000, le martèlement du thème du travail clandestin des Asiatiques avec l'invention du concept des "appartements raviolis" maintes fois objet de reportages télévisés où l'on transpose dans les appartements parisiens l'image les populations grouillantes et laborieuses des rizières. Les nouvelles représentations s'appuient donc sur un sédiment ancien, les stéréotypes peuvent donc se déplacer du négatif au positif suivant le contexte socio-économique, mais trouvent toujours à leur fondement la frontière que l'on établit entre soi et les autres. Si un sentiment de méfiance (on parle de "mystère", on évoque "une communauté énigmatique") naît dans les années 1980, les Asiatiques sont toujours considérés comme bien intégrés : l'enquête d'opinion de la SOFRES de 1984 montre que les Asiatiques ont un indice d'intégration très positif (+22) les plaçant juste derrière les immigrants d'Europe et bien devant les Africains noirs (-12) ou les Algériens (-49). De même, selon l'enquête de l'institut CSA de 1993, ils continuent à bénéficier d'un "bon indice de sympathie". Le tournant des années 1980 n'a donc pas entièrement effacé les stéréotypes favorables qui constituent comme un sédiment sur lequel se superposent d'autres représentations. Doan Bui, a reçu le prix Albert Londres qui récompense son travail de journaliste en 2013 et son livre récent Le Silence de mon père bénéficie d'une large couverture médiatique ${ }^{19}$. La qualité de son travail n'est pas discutable. C'est à un autre niveau, celui de la place occupée par les migrants vietnamiens et leurs descendants dans la sociologie des acteurs de l'industrie des médias, que la recherche en histoire de l'immigration peut revêtir un intérêt.

\section{La fabrique du "bon immigré"}

Lélite de la communauté asiatique, formée par des cadres diplômés, travaille à contrer le retournement d'opinion constaté plus haut. Michèle Guillon et Taboada Leonettijo montrent son rôle dans la structuration et la représentation des Chinois du XIII arrondissement de Paris. Pour Félix Wu, candidat aux municipales de 2007 dans cet arrondissement, il est urgent de valoriser sa communauté car " elle est mal connue ${ }^{21}$ ". Cette approche communautariste surfe sur la vague de la discrimination positive et revendique une visibilité politique pour les Asiatiques à l'instar des Arabes ou des Noirs. C'est également dans cette direction qu'il faudra analyser la naissance du récent Mouvement des citoyens français d'origine vietnamienne $(\mathrm{MCFV})^{22}$. Des immigrants veulent ainsi se donner à voir en cultivant leurs particularismes.

Force est de constater qu'ils sont aussi les agents de la formation de leurs propres stéréotypes par un procédé d'usurpation qui fait passer leur identité singulière pour une identité collective et communautaire. Ce processus n'est pas nouveau.

18. Pierre-Jean Simon, "L'Indochine française. Bref aperçu de son histoire et des représentations coloniales", in Hommes et Migrations, $n^{\circ}$ 1234, 2001, pp. 14-22. 19. Doan Bui, Le silence de mon père, Paris, L'Iconoclaste, 2016, 272 p. 20. Michelle Guillon et d'Isabelle Taboada Leonetti, Le Triangle de Choisy, un quartier chinois à Parisi, op. cit. 21. Félix Wu, "La communauté asiatique en France, une image à redéfinir", in Revue internationale et stratégique, vol. 1, n 73, 2009, pp. 113-116. 22. Extrait de sa charte de 2015 : “Ayant la volonté de rassembler le plus largement possible pour constituer un groupe d'influence incontournable auprès des institutions de la République, le M.C.F.V. sera un Mouvement d'influence..." 
Les recherches sur les Vietnamiens en France au sortir de la Seconde Guerre mondiale montrent, alors que les étudiants ne représentaient qu'une partie des Vietnamiens en France, et que les travailleurs et tirailleurs - majoritaires - étaient pour la plupart des paysans analphabètes, qu'il a existé le mythe vivace présentant le Vietnamien comme "intellectuel, intelligent et studieux". Ainsi, alors qu'ils étaient largement majoritaires, les travailleurs et les tirailleurs ont laissé les étudiants être leurs porte-parole à la tête de la Délégation des Indochinois, fondée en 1944 pour représenter les 25000 Indochinois résidant en France. La différence est qu'aujourd'hui ces "sans-voix" se rendent de plus en plus visibles comme on peut le voir à travers les manifestations des "sans-papiers". II faut d'ailleurs noter que ces derniers bénéficient davantage de la solidarité d'associations comme Réseau éducation sans frontière (RESF) que de soutien des associations "communautaires". L'élite garde ses distances. L'analyse de la construction de la "bonne image" permet de mettre en évidence le mouvement convergent auquel participent les immigrants eux-mêmes, soucieux de leur image, gage de leur accueil, et les acteurs de la formation de l'opinion dans la société d'accueil. Pour les premiers, la représentation par les élites leur assure une vision positive, leur démarquage avec les autres immigrés avec lesquels ils refusent d'être comparés ${ }^{23}$. Pour les autres, élites aussi de la société d'accueil (politiques, journalistes concourant à la formation de l'opinion publique), l'existence même de "bons immigrés" a une fonction normative permettant de définir par opposition ce qu'est un "mauvais immigré". Les Polonais et les Italiens avaient déjà tenus tenu ce rôle. Ceci a permis de nourrir les discours établissant une frontière entre les étrangers et les coloniaux, entre les immigrants européens et les autres. Les uns pour défendre l'idée que ces derniers seraient "inassimilables", les autres pour dénoncer la discrimination spécifique, dans un continuum colonial, dont ils seraient victimes. L'intégration de la "dimension asiatique" de l'immigration en France pourrait aider à dépasser les termes de ce débat. 